Check for updates

Cite this: RSC Adv., 2017, 7, 21790

Received 27th December 2016 Accepted 12th April 2017

DOI: $10.1039 / c 6 r a 28731 j$

rsc.li/rsc-advances

\section{Effects of Rh-doping on the photooxidative degradation activity of titanate nanosheets $\uparrow$}

\author{
Wasusate Soontornchaiyakul, (D) *a Takuya Fujimura, ${ }^{a}$ Hisanao Usami ${ }^{b}$ \\ and Ryo Sasai iD a
}

To investigate the Rh-doping effect on the photooxidative degradation activity of titanate nanosheets prepared by exfoliation of an $\mathrm{H}_{2} \mathrm{Ti}_{3} \mathrm{O}_{7}$ crystal in aqueous media, a colloidal aqueous suspension of titanate nanosheets doped with $\mathrm{Rh}$ atoms at the Ti sites (TiNS:Rhz, $z=$ amount of $\mathrm{Rh},\left[\mathrm{Ti}_{3-z} \mathrm{Rh}_{z} \mathrm{O}_{7}\right]^{2-}$ ) was prepared. Oxidative degradation of methylene blue (MB) by TiNS:Rhz in aqueous media under UV light irradiation was studied. Using X-ray diffraction, diffuse reflection spectroscopy, and photoelectrochemical measurements, the electronic band structure of TiNS:Rhz was elucidated and was shown not to change with the amount of Rh doping at the Ti sites. However, the degradation of MB increased with an increase in Rh doping. From these results, it is suggested that the redox reaction between $\mathrm{Rh}^{3+}$ and $\mathrm{Rh}^{4+}$ strongly contributes to the oxidative degradation of $\mathrm{MB}$ in the presence of TiNS:Rhz under UV light irradiation.

\section{Introduction}

Two-dimensional titanate nanosheets (TiNSs), which can be prepared from layered titanate, $\mathrm{H}_{2} \mathrm{Ti}_{n} \mathrm{O}_{2 n+1}(n \geq 3)$, and/or lepidocrocite-type titanate, $\mathrm{H}_{n} \mathrm{Ti}_{2-n / 4} \square_{n / 4} \mathrm{O}_{4}(n \sim 0.7 ; \square$, vacancy), have attracted attention because they exhibit unique structural features such as high aspect ratios (thickness: $1 \mathrm{~nm}$, surface area: $\sim$ several $\mu \mathrm{m}^{2}$ ) and malleability. Furthermore, they show useful physicochemical properties such as high cationexchangeability, wide bandgap semiconductor properties, high dielectric constant, and photocatalytic ability. ${ }^{1-5}$ Thus, TiNSs have been studied by many researchers as a gas barrier, in water purification, as a functional coating reagent, as a selfcleaning material, and so on. ${ }^{6,7}$ However, the photocatalytic activity of these nanosheets is not sufficient for their application as a self-cleaning material. Thus, improvement in their photocatalytic activity is desired.

The metal doping method, in which other metal atoms are incorporated into the $\mathrm{Ti}$ sites of titanate, is a well-known method to improve photocatalytic activity. Improvement using this method originates from the formation of a new energy

\footnotetext{
${ }^{a}$ Interdisciplinary Graduate School of Science and Engineering, Shimane University, 1060, Nishi-kawatsu-cho, Matsue, 690-8501, Shimane, Japan.E-mail: rsasai@riko. shimane-u.ac.jp; Fax: +81-852-32-6402 ext. 6103; Tel: +81-852-32-6402 ext. 6103 ${ }^{b}$ Faculty of Textile Science and Technology, Shinshu University, 3-15-1, Tokida, Ueda, 386-0018, Nagano, Japan

$\dagger$ Electronic supplementary information (ESI) available: The schematic of $\mathrm{H}_{2} \mathrm{Ti}_{3} \mathrm{O}_{7}$ crystal structure and their lattice parameters, the XRD patterns of Rh-doped sodium titanate, $\mathrm{Na}_{2} \mathrm{Ti}_{3-x} \mathrm{Rh}_{x} \mathrm{O}_{7}, \mathrm{H}_{2} \mathrm{Ti}_{3-x} \mathrm{Rh}_{x} \mathrm{O}_{7}$, and non-exfoliated TiNS:Rhz, the AFM of TiNS:Rhz, and XPS data for Ti and Rh compared between TiNS: $\mathrm{Rh}_{0}$ and TiNS:Rh ${ }_{10}$. See DOI: 10.1039/c6ra28731j
}

band by orbital mixing and change in the carrier concentration. ${ }^{\mathbf{8} 9}$ Adjustment of the photocatalytic properties of titanate affects its photoredox activity.

Recently, there have been many investigations into the metal doping of TiNSs. Ida et al. reported the photocatalytic activity of Rh-doped, lepidocrocite-type TiNSs, prepared by the exfoliation of $\mathrm{H}_{n} \mathrm{Ti}_{2-n / 4} \square_{n / 4} \mathrm{O}_{4}\left(n \sim 0.7 ; \square\right.$, vacancy). ${ }^{10}$ In this work, it was reported that $\mathrm{Rh}$ atoms incorporated into the $\mathrm{Ti}$ sites of the TiNSs acted as reaction sites for photo-induced water splitting. Milanović et al. also reported the synthesis of Nb-doped, lepidocrocite-type TiNSs and the effect of $\mathrm{Nb}$ doping on the photodegradation of methylene blue (MB) in water purification. ${ }^{11}$ Furthermore, Sasaki et al. reported the synthesis of lepidocrocite-type $\mathrm{Ti}_{0.6} \mathrm{Fe}_{0.4} \mathrm{O}_{2}$ and $\mathrm{Ti}_{0.8} \mathrm{Co}_{0.2} \mathrm{O}_{2}$ nanosheets. ${ }^{12}$ It was shown that doping $3 \mathrm{~d}$ metals into the Ti sites affected the crystal and electronic structures of the lepidocrocite-type TiNSs. The metal doping and effect of doping on the photocatalytic properties of TiNSs prepared by exfoliation of an $\mathrm{H}_{2} \mathrm{Ti}_{n} \mathrm{O}_{2 n+1}$ crystal have not been reported to date.

$\mathrm{H}_{2} \mathrm{Ti}_{3} \mathrm{O}_{7}$ crystal can be prepared from layered $\mathrm{Na}_{2} \mathrm{Ti}_{3} \mathrm{O}_{7}$ by the $\mathrm{Na}^{+} / \mathrm{H}^{+}$ion-exchange. The basic layer architecture of $\mathrm{H}_{2} \mathrm{Ti}_{3} \mathrm{O}_{7}$ was maintained nearly unchanged from that in $\mathrm{Na}_{2} \mathrm{Ti}_{3} \mathrm{O}_{7}$ which the structure was determined by Andersson and Wadsley. ${ }^{13} \mathrm{Na}_{2} \mathrm{Ti}_{3} \mathrm{O}_{7}$ consists of edge-shared and corner-shared $\mathrm{TiO}_{6}$ octahedral, which the sheet layers are separated by the $\mathrm{Na}^{+}$ ion (as shown in Fig. S1 in ESI $\dagger$ ). Unlike the lepidocrocite-type titanate, $\mathrm{H}_{n} \mathrm{Ti}_{2-n / 4} \square_{n / 4} \mathrm{O}_{4}\left(n \sim 0.7 ; \square\right.$, vacancy), the $\mathrm{H}_{2} \mathrm{Ti}_{3} \mathrm{O}_{7}$ crystal have no vacancies in their basic layer architecture. However, it is possible to dope some kinds of metals in Ti site such as $\mathrm{Nb}, \mathrm{Rh}, \mathrm{Fe}, \mathrm{Al}$, and etc. which their ionic radius not much different compared to Ti. 
In this study, Rh-doped TiNSs (TiNS:Rhz, $z$ is the amount of Rh) were synthesised as a $\mathrm{Na}_{2} \mathrm{Ti}_{3-x} \mathrm{Rh}_{x} \mathrm{O}_{7}$ crystal by solid-state reaction. The electronic band structure of the synthesised TiNS:Rhz was characterized by photocurrent and photoabsorption measurements. The photodegradation of MB in the presence of TiNS:Rhz was investigated to evaluate its photocatalytic activity.

\section{Experimental}

\subsection{Reagents and materials}

Sodium carbonate $\mathrm{Na}_{2} \mathrm{CO}_{3}$ (99.9\%) from Wako Pure Chemical Industries Co., anatase-type $\mathrm{TiO}_{2}(99.7 \%)$ from High Purity Chemicals Co., $\mathrm{Rh}_{2} \mathrm{O}_{3}$ (99.9\%) from Wako Pure Chemical Industries Co., methylamine $\left(\mathrm{CH}_{3} \mathrm{NH}_{2}, 40 \mathrm{wt} \%\right.$ solution) and tetramethylammonium hydroxide (TMAOH, $26 \mathrm{wt} \%$ solution) from Tokyo Chemical Industry Co., and $\mathrm{MB}\left(\mathrm{C}_{16} \mathrm{H}_{18} \mathrm{~N}_{3^{-}}\right.$ $\mathrm{SCl} \cdot 3 \mathrm{H}_{2} \mathrm{O}, \quad 3,7$-bis(dimethylamino)-phenothiazin-5-ium chloride) from Kanto chemical Co. were used as received.

\subsection{Synthesis of Rh-doped sodium titanate}

$\mathrm{Na}_{2} \mathrm{Ti}_{3-x} \mathrm{Rh}_{x} \mathrm{O}_{7}$ was synthesized using a modified form of the solid-state reaction method reported by Izawa et al. $^{\mathbf{1 4}}$ as the following procedures. $\mathrm{Na}_{2} \mathrm{CO}_{3}$, anatase-type $\mathrm{TiO}_{2}$ and $\mathrm{Rh}_{2} \mathrm{O}_{3}$ were mixed and grinded in a mortar. The mixture was calcined at 1173 $\mathrm{K}$ for $24 \mathrm{~h}$ in air. This operation was repeated again after grinding. The amount of $\operatorname{Rh}(z)$ added to the Ti sites was set from 0 to $10 \%$.

\subsection{Preparation of the nanosheet colloidal suspension}

The colloidal suspension of TiNS:Rhz was prepared according to a modified form of the method described by Miyamoto et $\mathrm{al}{ }^{15}$ $0.3 \mathrm{~g}$ of $\mathrm{Na}_{2} \mathrm{Ti}_{3-x} \mathrm{Rh}_{x} \mathrm{O}_{7}$ powder was dispersed in $30 \mathrm{~cm}^{3}$ of hydrochloric acid $\left(1 \mathrm{~mol} \mathrm{dm}^{-3}\right)$ for exchanging the counter cation from $\mathrm{Na}^{+}$to $\mathrm{H}^{+}$. Subsequently, it was shaken for 3 days at room temperature. The hydrochloric acid was replaced every day to allow the protonation reaction to proceed effectively. The filtrated $\mathrm{H}_{2} \mathrm{Ti}_{3-x} \mathrm{Rh}_{x} \mathrm{O}_{7}$ was washed with high purity water to remove any remaining hydrochloric acid, and was dried under a reduced pressure condition at room temperature overnight. The collected $\mathrm{H}_{2} \mathrm{Ti}_{3-x} \mathrm{Rh}_{x} \mathrm{O}_{7}$ was neutralized by methylamine aqueous solution at $333 \mathrm{~K}$ for 6 days to yield $\left(\mathrm{CH}_{3} \mathrm{NH}_{3}\right)_{2}$ $\mathrm{Ti}_{3-x} \mathrm{Rh}_{x} \mathrm{O}_{7}$ powder. Subsequently, $\left(\mathrm{CH}_{3} \mathrm{NH}_{3}\right)_{2} \mathrm{Ti}_{3-x} \mathrm{Rh}_{x} \mathrm{O}_{7}$ was dispersed in TMAOH solution $\left(\left[\mathrm{Ti}_{3-x} \mathrm{Rh}_{x} \mathrm{O}_{7}{ }^{2-}\right] /[\mathrm{TMAOH}]=5\right)$ by sonication for 5 days. Non-exfoliated TiNS:Rhz was removed by centrifugation (at $4000 \mathrm{rpm}$ or $1150 \mathrm{~g}$ for $15 \mathrm{~min}$, IEC61010-2020, KUBOTA). The TiNS:Rhz colloidal suspension was obtained as the supernatant.

\subsection{Adsorption experiment}

The adsorption experiment of $\mathrm{MB}^{\mathbf{1 6 - 1 8}}$ by TiNS:Rhz was carried out by the following procedure: (1) $0.01 \mathrm{~cm}^{3}$ of the TiNS:Rhz $\left(9.53 \times 10^{-2} \mathrm{~mol} \mathrm{dm}{ }^{-3}, \mathrm{pH} 11\right)$ was added to $10 \mathrm{~cm}^{3}$ of the $\mathrm{MB}$ aqueous solution $\left(9.53 \times 10^{-8}\right.$ to $\left.9.53 \times 10^{-5} \mathrm{~mol} \mathrm{dm}^{-3}, \mathrm{pH} \mathrm{11}\right)$, and this mixture was stirred at room temperature for $24 \mathrm{~h}$, away from light. The molar ratio of the MB and TiNS:Rh $z$ was varied from 0.001 to 1.0 . (2) After stirring, the mixture was filtrated through a hydrophilic polyfluororesin membrane filter (pore size: $0.1 \mu \mathrm{m}$ ) to remove TiNS:Rhz with MB from the suspension. (3) The amount of adsorbed MB by TiNS:Rhz was calculated from the absorbance of $\mathrm{MB}$ in the filtrate at the peak wavelength of $663 \mathrm{~nm}$. The extinction coefficient $(\varepsilon)$ of the MB solution $(\mathrm{pH}$ 11) was $5.2 \times 10^{4} \mathrm{dm}^{3} \mathrm{~mol}^{-1} \mathrm{~cm}^{-1}$.

\subsection{UV light irradiation experiment}

To characterize the photocatalytic activity of TiNS:Rhz in aqueous media, the solution of TiNS:Rhz and MB was irradiated with UV light according to the following procedure: (1) the MB solution $\left(4.7 \times 10^{-6} \mathrm{~mol}\right)$ was mixed with TiNS:Rhz $\left(4.7 \times 10^{-6} \mathrm{~mol}\right)$ to reach a total volume of $10 \mathrm{~cm}^{3}(\mathrm{pH} \mathrm{11})$, and stirred in the dark until the adsorption was at equilibrium. (2) UV light of $254 \mathrm{~nm}\left(0.88 \mathrm{~mW} \mathrm{~cm}^{-2}\right)$ from a Xe lamp (MAX-301, Asahi Spectra Co.) was directed towards the mixture through a band pass filter, for a given time at room temperature. The irradiation area was confined to a square of $25 \mathrm{~cm}^{2}$. (3) After the UV irradiation, the mixture was filtrated by the hydrophilic polyfluororesin membrane filter (pore size: $0.1 \mu \mathrm{m}$ ) to remove TiNS:Rhz from the suspension. (4) The amount of adsorbed MB by TiNS:Rhz was calculated from the absorbance of MB in the filtrate at the peak wavelength of $663 \mathrm{~nm}$.

\subsection{Characterization}

X-ray diffraction (XRD) patterns were obtained by an X-ray diffractometer (RIGAKU, MiniFlex II) with Ni-filtered CuKa radiation $(30 \mathrm{kV}, 15 \mathrm{~mA})$. The amount of $\mathrm{Rh}$ in TiNS:Rhz was determined by inductively coupled plasma-atomic emission spectroscopy (ICP-AES: Optima -2000, Perkin-Elmer) after fully dissolving the prepared TiNS:Rhz in nitric acid $\left(1.0 \mathrm{~mol} \mathrm{dm}^{-3}\right)$. Absorption spectra of various samples were measured by a UVVis spectrophotometer (V-670, JASCO). Diffuse reflection spectra of the $\mathrm{H}_{2} \mathrm{Ti}_{3-x} \mathrm{Rh}_{x} \mathrm{O}_{7}$ powders were measured using the UV-Vis spectrophotometer (V-670, JASCO) attached to an integrating sphere system (ISN-723, JASCO).

According to the measurement setup reported by Usami et al. ${ }^{19,20}$ photocurrent was measured by a three electrode electrochemical analysing system (Hokuto Denko, HSV-110) at a sweep rate of $10 \mathrm{mV} \mathrm{s}^{-1}$. The TiNS:Rhz was cast on FTO glass and dried at $333 \mathrm{~K}$ overnight. The TiNS:Rhz film deposited on FTO glass acted as the working electrode, which was placed on a circle ( $9 \mathrm{~mm}$ in diameter) in a PTFE cell to adjust the electrode area to $0.636 \mathrm{~cm}^{2}$. $\mathrm{An} \mathrm{Ag} / \mathrm{AgNO}_{3}$ reference electrode and Pt wire counter electrode were immersed in an acetonitrile solution of $0.1 \mathrm{~mol} \mathrm{dm}^{-3}$ tetraethylammonium perchlorate. A parallel beam of excitation light from a $150 \mathrm{~W}$ Xe lamp (Hamamatsu Photonics, L2195) was focused on the semiconductor layer of the FTO electrode through a light chopper (NF Electric Instruments, 5584A). The modulated photocurrent at the applied potential was amplified using a digital lock-in-amplifier (NF Electric Instruments, LI5640) and transferred to a PC to be plotted against the applied potential. 


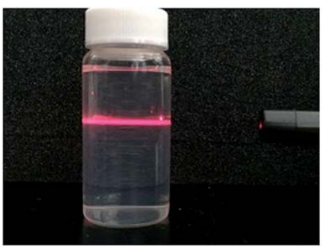

Fig. 1 The Tyndall effect observed by passing a laser beam through the TiNS: Rhz colloidal suspension $\left(\sim 0.5 \mathrm{mmol} \mathrm{dm}^{-3}\right)$.

\section{Results and discussion}

\subsection{Characterization of TiNS:Rhz}

Fig. 1 is a photograph of the prepared TiNS:Rhz colloidal suspension, which is transparent but shows scattering of an incident laser beam because of the Tyndall effect. It was found that TiNS:Rhz from $\mathrm{Na}_{2} \mathrm{Ti}_{3-x} \mathrm{Rh}_{x} \mathrm{O}_{7}$ could be exfoliated in all of the suspensions by the described procedures. The chemical compositions of the prepared TiNS:Rhz are shown in Table 1. The actual amount of Rh in TiNS:Rhz was lower that what was initially added. Some $\mathrm{Rh}$ may have been removed during protonation, intercalation of methylamine, or exfoliation by TMAOH (see Fig. S2 and S3 in ESI $\dagger$ ). In the XRD patterns of the cast films prepared from the TiNS:Rhz colloidal suspension, diffraction peaks originating from the deposited unknown crystal compounds were not found, since the deposited unknown crystal compounds were not layered materials and could not be exfoliated by the TMAOH aqueous solution.

Fig. 3 shows the UV-visible absorption spectra of all the TiNS:Rhz colloidal suspensions of the same concentration 1 $\left(2 \mathrm{mg} \mathrm{dm}{ }^{-3}\right.$ ). The band edge originates from the band gap of the TiNS:Rhz, which exhibits a blue shift with respect to the diffuse reflectance spectra of the $\mathrm{H}_{2} \mathrm{Ti}_{3} \mathrm{O}_{7}$ powder. This blue shift can be explained by the quantum-size effect. ${ }^{21,22}$ The TiNS:Rhz existed as nanosheets with a thickness about $1.5 \mathrm{~nm}$ in the colloidal suspension (see Fig. S4 in ESI $\dagger$ ). The optical band gap $\left(E_{\mathrm{g}}\right.$, estimated from the UV-vis absorption spectra of the TiNS:Rhz colloidal suspension) and the conduction band level ( $E_{\mathrm{CB}}$, estimated from the photocurrent measurement of the TiNS:Rhz cast film) are shown in Table 1 . Both $E_{\mathrm{g}}$ and $E_{\mathrm{CB}}$ of all samples were not influenced by Rh doping into the Ti sites of the lattice.

\subsection{The adsorption of methylene blue molecules by TiNS:Rhz}

The adsorption of MB by TiNS:Rhz was evaluated by plotting the molar amount of adsorbed $\mathrm{MB}$ per $\left[\mathrm{Ti}_{3-x} \mathrm{Rh}_{x} \mathrm{O}_{7}{ }^{2-}\right]$ unit, $C^{\mathrm{abs}}$

Table 1 The chemical formula, optical band gap $\left(E_{g}\right)$, and conduction band potential $\left(E_{\mathrm{CB}}\right)$ of the TiNS:Rhz samples

\begin{tabular}{llll}
\hline Sample & Chemical formula & $E_{\mathrm{g}}(\mathrm{eV})$ & $\begin{array}{l}E_{\mathrm{CB}}(\mathrm{V} \\
\text { vs. NHE })\end{array}$ \\
\hline TiNS:Rh0 & {$\left[\mathrm{Ti}_{3} \mathrm{O}_{7}\right]^{2-}$} & $4.20 \pm 0.04$ & 0.00 \\
TiNS:Rh1 & {$\left[\mathrm{Ti}_{2.999} \mathrm{Rh}_{0.001} \mathrm{O}_{7}\right]^{(2+\delta)-}$} & $4.17 \pm 0.04$ & 0.00 \\
TiNS:Rh2 & {$\left[\mathrm{Ti}_{2.992} \mathrm{Rh}_{0.008} \mathrm{O}_{7}\right]^{(2+\delta)-}$} & $4.17 \pm 0.04$ & 0.00 \\
TiNS:Rh5 & {$\left[\mathrm{Ti}_{2.970} \mathrm{Rh}_{0.030} \mathrm{O}_{7}\right]^{(2+\delta)-}$} & $4.15 \pm 0.04$ & -0.01 \\
TiNS:Rh10 & {$\left[\mathrm{Ti}_{2.952} \mathrm{Rh}_{0.048} \mathrm{O}_{7}\right]^{(2+\delta)-}$} & $4.18 \pm 0.04$ & -0.01
\end{tabular}

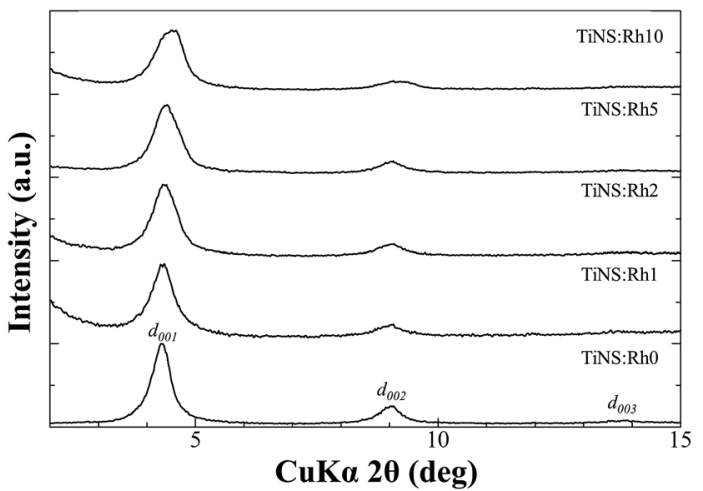

Fig. 2 XRD patterns of TiNS:Rhz films cast on quartz substrates.

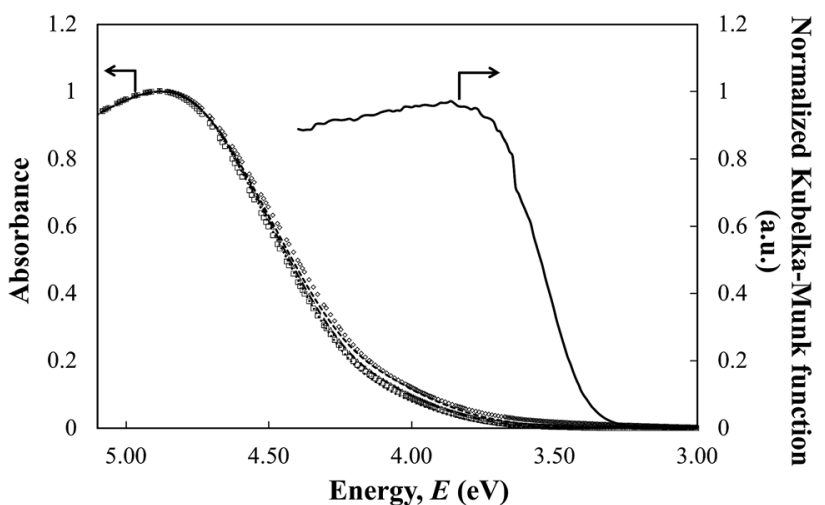

Fig. 3 The UV-vis absorption spectra of the TiNS:Rhz colloidal suspension (concentration of $2 \mathrm{mg} \mathrm{dm}^{-3}$ ) and diffuse reflectance spectra of the $\mathrm{H}_{2} \mathrm{Ti}_{3} \mathrm{O}_{7}$ powders. ((-) TiNS:Rh0, $(\Delta)$ TiNS:Rh1, $(-\bullet)$ TiNS:Rh2, ( $\diamond)$ TiNS:Rh5, ( $\square)$ TiNS:Rh10, and (-) $\left.\mathrm{H}_{2} \mathrm{Ti}_{3} \mathrm{O}_{7}\right)$.

(mol $\mathrm{mol}^{-1}$ ) against the molar amount of added $\mathrm{MB}$ per $\left[\mathrm{Ti}_{3-x} \mathrm{Rh}_{x} \mathrm{O}_{7}{ }^{2-}\right]$ unit, $C^{0}$ (mol mol ${ }^{-1}$ ), as shown in Fig. 4. The value of $C^{\text {abs }}$ increased along with an increase in the $C^{0}$ value. Therefore, the amount of adsorbed $\mathrm{MB}$ at saturation point could be calculated, and this is shown in Table 2 . The adsorbed

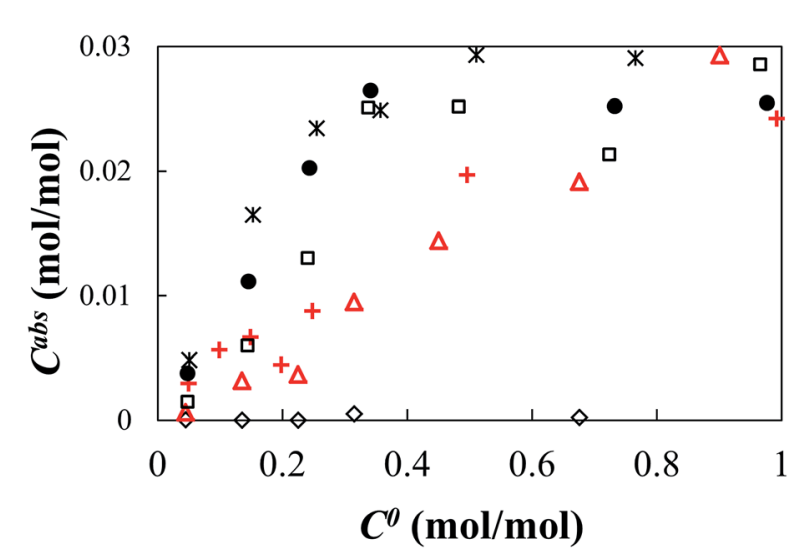

Fig. 4 The adsorption isothermal curves of MB by TiNS:Rhz. (( $\diamond)$ Absence of TiNS:Rhz, (+) TiNS:Rh0, $(\Delta)$ TiNS:Rh1, $(\square)$ TiNS:Rh2, $(x)$ TiNS:Rh5, and (-) TiNS:Rh10). 
Table 2 Amount of absorbed MB by TiNS:Rhz

\begin{tabular}{ll}
\hline Sample & $\begin{array}{l}\text { Saturated amount of absorbed MB } \\
\text { per molar amount of TiNS:Rhz } \\
\text { at equilibrium }\left(\mathrm{mg} \mathrm{g}^{-1}\right)\end{array}$ \\
\hline TiNS:Rh0 & $37 \pm 3$ \\
TiNS:Rh1 & $36 \pm 3$ \\
TiNS:Rh2 & $35 \pm 3$ \\
TiNS:Rh5 & $31 \pm 3$ \\
TiNS:Rh10 & $29 \pm 3$
\end{tabular}

amount was much lower than the formal anionic charge of TiNS:Rhz $\left(2 \mathrm{~mol} \mathrm{~mol}^{-1}\right)$ in all cases because of the competitive adsorption of MB and TMAOH. Table 2 also shows that the amount of adsorbed MB slightly decreased with an increase in Rh doping. This can be explained by the anionic charge of TiNS:Rhz. In the case of TiNS:Rhz $(z>0)$, the anionic charge changed because the ionic state of $\mathrm{Rh}$ was $\mathrm{Rh}^{3+}$ or $\mathrm{Rh}^{4+}$. This lead to an increase in the anionic charge of TiNS:Rhz. However, the result did not affect the MB adsorption because it strongly interacted with the TMA cation. As a result, the adsorption of MB decreased when Rh was incorporated into the Ti sites.

The shape of the adsorption isothermal curve was different for each sample. The $C^{\text {abs }}$ value of the TiNS:Rh0 and TiNS:Rh1 linearly increased with an increase in the $C^{0}$ value. The adsorption isothermal curve of TiNS:Rh1 behaved similarly to TiNS:Rh0 but the $C^{\text {abs }}$ value of TiNS:Rh2, TiNS:Rh5 and TiNS:Rh10 logarithmically increased. Thus, these results suggest that the surface properties of TiNS varied with Rh doping into the Ti sites.

\subsection{Photodegradation of methylene blue}

Degradation of MB by UV light irradiation was observed by changes in its UV-visible absorption spectra. Fig. 5 shows the UV-visible absorption spectra of filtrated MB solution from the mixture with TiNS:Rh0 after irradiation by UV light with a wavelength of $254 \mathrm{~nm}$, at different irradiation times. The spectra of the solution were similar with a gradual decrease in absorbance seen for the peak at around $663 \mathrm{~nm}$, originating from MB. This was observed for all TiNS:Rhz samples, which

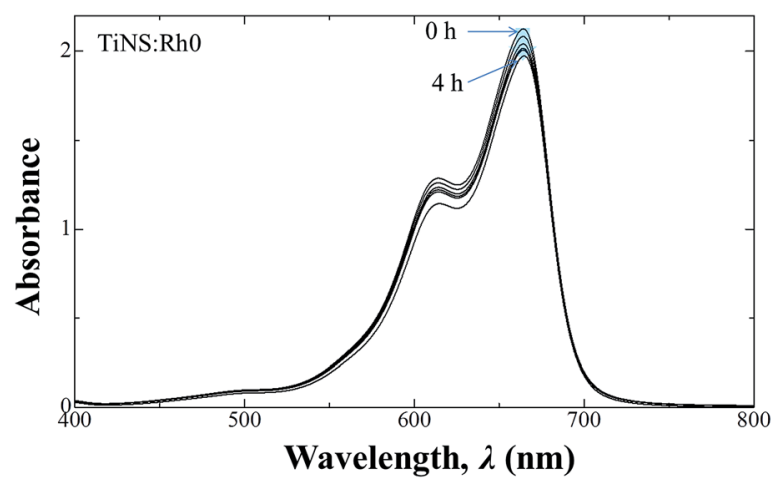

Fig. 5 UV-vis absorption spectral changes of MB solution under UV light (at a wavelength of $254 \mathrm{~nm}$ ) irradiation in the presence of TiNS:RhO.

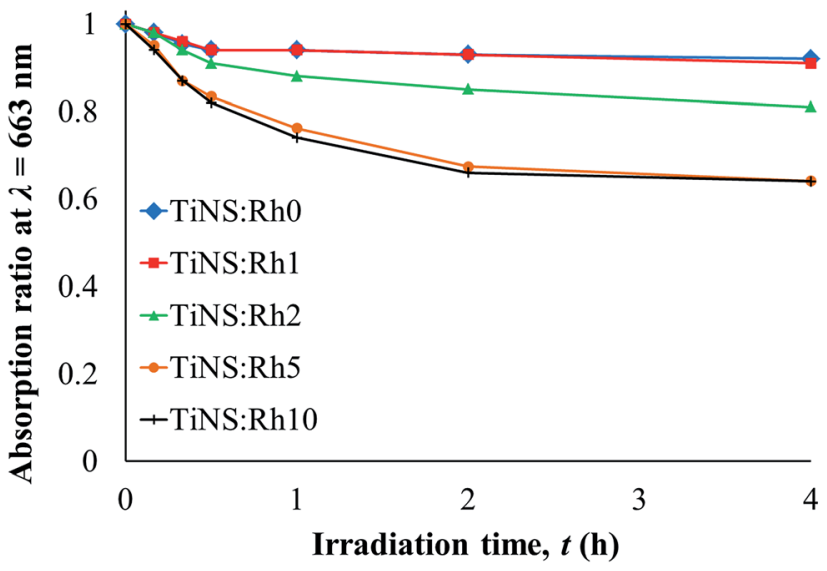

Fig. 6 The photodegradation ratio curves of the maximum absorption band of MB in the presence of the different TiNS:Rhz samples (at 663 $\mathrm{nm})$, after the UV light $(\lambda=254 \mathrm{~nm})$ irradiation over different irradiation times.

shows that the MB was degraded by UV light irradiation and that TiNS:Rhz acted as a photocatalyst to decompose MB.

To compare photocatalytic activity of each TiNS:Rhz series, the absorbance ratio values at $663 \mathrm{~nm}\left(\mathrm{Abs}_{t} / \mathrm{Abs}_{0}\right.$; where $\mathrm{Abs}_{t}$ is an absorbance at each interval and $\mathrm{Abs}_{0}$ is the initial absorbance or absorbance at $t=0$ ) are plotted against the irradiation time in Fig. 6. All TiNS:Rhz samples photocatalytically degraded MB. However, the duration of the reaction varied. TiNS:Rh0 and TiNS:Rh1 showed almost the same photodegradation ratio curve, while TiNS:Rh2, TiNS:Rh5, and TiNS:Rh10 produced a different result. This indicates that photocatalytic activity can be improved by Rh doping at the Ti sites of TiNS:Rhz.

The quantum yield $\left(\Phi_{\mathrm{deg}}\right)$ of the photodegradation reaction of MB by TiNS:Rhz at initial time was calculated by:

$$
\Phi_{\text {deg }}=\frac{\text { number of degraded MB molecules }}{\text { number of incident photons }}
$$

Table 3 shows the $\Phi_{\text {deg }}$ value of each TiNS:Rhz sample. TiNS:Rh0 and TiNS:Rh1 exhibit almost the same $\Phi_{\text {deg }}$ value. In contrast, the $\Phi_{\text {deg }}$ value of TiNS:Rh2, TiNS:Rh5, and TiNS:Rh10 are higher than that of TiNS:Rh0; thus, $\Phi_{\text {deg }}$ increased with increasing Rh doping. A similar divide between samples was observed in the adsorption study (see Table 2). Thus, it is evident that $\mathrm{Rh}$ doping modifies the surface properties of TiNS:Rhz. However, the adsorption ability was not improved with an increase in Rh doping. This may be because the size of TiNS:Rhz decreases with increase in Rh doping (see Fig. 2).

Table 3 Quantum yield $\left(\Phi_{\mathrm{deg}}\right)$ of MB photodegradation

\begin{tabular}{ll} 
Sample & $\Phi_{\text {deg }}(\%)$ \\
\hline TiNS:Rh0 & 0.30 \\
TiNS:Rh1 & 0.31 \\
TiNS:Rh2 & 0.34 \\
TiNS:Rh5 & 0.81 \\
TiNS:Rh10 & 0.97
\end{tabular}




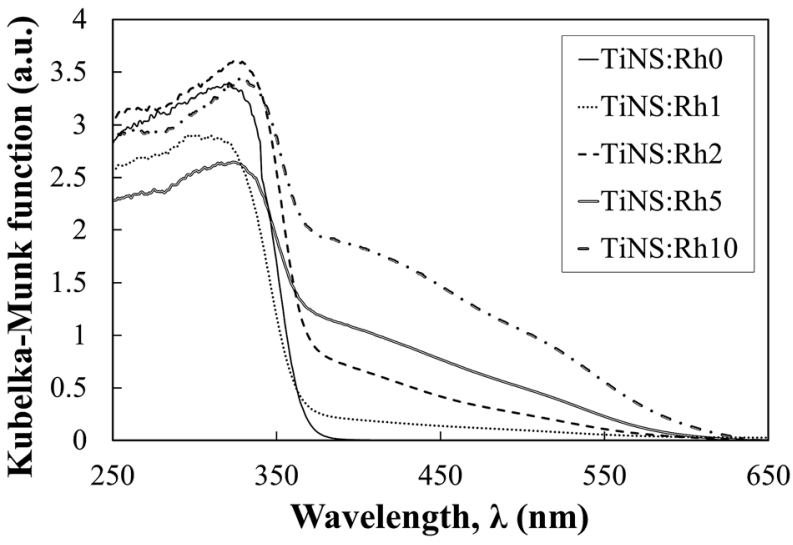

Fig. 7 The diffuse reflection spectra of the $\mathrm{H}_{2} \mathrm{Ti}_{3-x} \mathrm{Rh}_{x} \mathrm{O}_{7}$ powders.

Moreover, the difference in photocatalytic ability cannot be explained by the energy band structure because the band gap energy and band edge potentials of the conduction and valence bands did not vary with Rh doping (see Table 1).

Fig. 7 shows the diffuse reflection spectra of the $\mathrm{H}_{2} \mathrm{Ti}_{3-x^{-}}$ $\mathrm{Rh}_{x} \mathrm{O}_{7}$ powders in terms of the Kubelka-Munk function. The absorption band edge at $350 \mathrm{~nm}$ originating from the band gap transition is observed in all of the TiNS:Rhz spectra. A broad absorption band appeared in the wavelength range from 350 to $600 \mathrm{~nm}$ when $\mathrm{Rh}$ atoms were incorporated into the Ti sites. The intensity of these new bands increased with an increase in Rh doping. According to previous results reported by Kudo et al., $\mathrm{Rh}^{3+}$ and $\mathrm{Rh}^{4+}$ incorporated into the Ti sites of $\mathrm{SrTiO}_{3}$ showed broad absorption bands around a wavelength of 400 and $500 \mathrm{~nm}$, respectively. ${ }^{23-27}$ Hence, Rh dopants in the present work existed as both $\mathrm{Rh}^{3+}$ and $\mathrm{Rh}^{4+}$. This also has been confirmed by measuring the X-ray photoelectron spectroscopy. As a result, the peak of $\mathrm{Rh} 3 \mathrm{~d}$ at $308.9 \mathrm{eV}$ and $309.9 \mathrm{eV}$ could be found, and thus this result show the existence of $\mathrm{Rh}^{3+}$ and $\mathrm{Rh}^{4+}$ (see also in Fig. S5 and S6 of ESI†). The different photocatalytic activities of TiNS:Rhz in MB photodegradation may be explained by the existence of both $\mathrm{Rh}^{3+}$ and $\mathrm{Rh}^{4+}$. Irradiation of TiNS:Rh0 by UV light is known to cause oxidative degradation of MB by photoinduced holes, $\mathrm{OH}$ radicals, and superoxide anions. ${ }^{28-31}$ The redox cycle of $\mathrm{Rh}^{3+}$ and $\mathrm{Rh}^{4+}$ in TiNS:Rhz $(z>0)$ can be driven by band gap excitation of TiNS under UV light irradiation due to a hole pool effect of Rh atoms in six fold coordination with the oxygen atoms. ${ }^{32}$ Thus, the improvement of photocatalytic activities of TiNS:Rhz can be caused by the redox cycle of the Rh cations $\left(\mathrm{Rh}^{3+}\right.$ and $\left.\mathrm{Rh}^{4+}\right)$ under UV light irradiation. However, it is still unclear whether the redox cycle of the Rh cations in the studied TiNS:Rhz samples was driven by the UV light. This will be the next challenging topic for research not only in titanate nanosheets but also in other metal oxide nanosheets and oxide materials.

\section{Conclusions}

In this study, the synthesis and photoelectrochemical characterization of TiNS:Rhz was performed. The band structure of the
TiNS:Rhz was not affected by Rh incorporation into the Ti sites. However, both adsorption and photodegradation behaviour of MB by TiNS:Rhz varied with Rh doping. The photodegradation activity of TiNS:Rhz was significantly improved with increased $\mathrm{Rh}$ doping, suggesting that the redox cycle of $\mathrm{Rh}^{3+}$ and $\mathrm{Rh}^{4+}$ plays an important role in the photodegradation of MB under UV light irradiation in the presence of TiNS:Rhz.

\section{Acknowledgements}

This research was partly supported by KAKENHI 24651144 from MEXT, Japan. The synchrotron radiation experiments were performed at the BL02B2 of SPring-8 with the approval of the Japan Synchrotron Radiation Research Institute (JASRI) (Proposal No. 2014B1744). Authors acknowledge the cooperation of Interdisciplinary Center for Science Research, Shimane University, for providing the experimental facility of SPM. We thank Dr. Yusuke DATE from Department of Materials Science, National Institute of Technology, Yonago College for X-ray photoelectron spectroscopy measurement.

\section{References}

1 H. Sato, K. Ono, T. Sasaki and A. Yamagishi, J. Phys. Chem. B, 2003, 107, 9824-9828.

2 T. Sasaki, Y. Ebina, M. Watanabe and G. Decher, Chem. Commun., 2000, 2163-2164.

3 T. Sasaki, Y. Ebina, Y. Kitami, M. Watanabe and T. Oikawa, J. Phys. Chem. B, 2001, 105, 6116-6121.

4 T. Sasaki, Y. Ebina, K. Fukuda, T. Tanaka, M. Harada and M. Watanabe, Chem. Mater., 2002, 14, 3524-3530.

5 T. Maluangnont, K. Matsuba, F. Geng, R. Ma, Y. Yamauchi and T. Sasaki, Chem. Mater., 2013, 25, 3137-3146.

6 X. Dong, M. Osada, H. Ueda, Y. Ebina, Y. Kotani, K. Ono, S. Ueda, K. Kobayashi, K. Takada and T. Sasaki, Chem. Mater., 2009, 21, 4366-4373.

7 M. Harada, T. Sasaki, Y. Ebina and M. Watanabe, $J$. Photochem. Photobiol., A, 2002, 148, 273-276.

8 T. Sasaki, Y. Ebina, Y. Kitami, M. Watanabe and T. Oikawa, J. Phys. Chem. B, 2001, 105, 6116-6121.

9 H. Sun, G. Zhou, S. Liu, H. M. Ang, M. O. Tadé and S. Wang, Chem. Eng. J., 2013, 231, 18-25.

10 S. Ida, N. Kim, E. Ertekin, S. Takenaka and T. Ishihara, J. Am. Chem. Soc., 2015, 137, 239-244.

11 M. Milanović, L. M. Nikolić, I. Stijepović, A. G. Kontos and K. P. Giannakopoulos, Mater. Chem. Phys., 2014, 148, 874881.

12 Y.-H. Kim, M. Osada, L. Dong, H.-J. Kim and T. Sasaki, J. Ceram. Soc. Jpn., 2015, 123, 335-339.

13 S. Andersson and A. D. Wadsley, Acta Crystallogr., 1961, 14, 1245-1249.

14 H. Izawa, S. Kikkawa and M. Koizumi, J. Phys. Chem., 1982, 86, 5023-5026.

15 N. Miyamoto, K. Kuroda and M. Ogawa, J. Mater. Chem., 2004, 14, 165.

16 W. Kuo and P. Ho, Dyes Pigm., 2006, 71, 212-217.

17 W. Kuo and P. Ho, Chemosphere, 2001, 45, 77-83. 
18 M. Feng, W. You, Z. Wu, Q. Chen and H. Zhan, ACS Appl. Mater. Interfaces, 2013, 5, 12654-12662.

19 H. Usami and S. Sasaki, J. Photopolym. Sci. Technol., 2013, 26, 207-211.

20 H. Usami, Y. Iijima, Y. Moriizumi, H. Fujimatsu, E. Suzuki and H. Inoue, Res. Chem. Intermed., 2007, 33, 101-110.

21 T. Sasaki, M. Watanabe, H. Hashizume, H. Yamada and H. Nakazawa, J. Am. Chem. Soc., 1996, 118, 8329.

22 M. Osada and T. Sasaki, Adv. Mater., 2012, 24, 209-228.

23 A. Kudo and Y. Miseki, Chem. Soc. Rev., 2009, 38, 253-278.

24 R. Konta, T. Ishii, H. Kato and A. Kudo, J. Phys. Chem. B, 2004, 108, 8992-8995.

25 K. Iwashina and A. Kudo, J. Am. Chem. Soc., 2011, 133, 13272-13275.
26 S. Kawasaki, K. Nakatsuji, J. Yoshinobu, F. Komori, R. Takahashi, M. Lippmaa, K. Mase and A. Kudo, Appl. Phys. Lett., 2012, 101, 033910.

27 S. Kawasaki, K. Akagi, K. Nakatsuji, S. Yamamoto, I. Matsuda, Y. Harada, J. Yoshinobu, F. Komori, R. Takahashi, M. Lippmaa, C. Sakai, H. Niwa, M. Oshima, K. Iwashina and A. Kudo, J. Phys. Chem. C, 2012, 116, 24445-24448.

28 A. Mills and J. Wang, J. Photochem. Photobiol., A, 1999, 127, 123-134.

29 R. Matthews, Water Res., 1991, 25, 1169-1176.

30 A. Houas, Appl. Catal., B, 2001, 31, 145-157.

31 K. Wetchakun, N. Wetchakun and S. Phanichphant, Desalin. Water Treat., 2015, 57, 10286-10294.

32 J. Kuncewicz and B. Ohtani, Chem. Commun., 2015, 51, 298301. 